\title{
Lung squamous cell carcinoma with rare epidermal growth factor receptor mutation G719X: a case report and literature review
}

\author{
Huanhuan Bi ${ }^{1 \#}$, Dunqiang Ren ${ }^{1 \#}$, Jieqiong $\mathrm{Wu}^{1}$, Xiaoqian Ding ${ }^{1}$, Caihong Guo ${ }^{1}$, Satoru Miura ${ }^{2}$, \\ Zsolt Megyesfalvi $^{3,4,5}$, Surein Arulananda ${ }^{6}$, Hongmei Wang ${ }^{1}$ \\ ${ }^{1}$ Department of Respiratory and Critical Care Medicine, The Affiliated Hospital of Qingdao University, Qingdao, China; ${ }^{2}$ Department of Internal \\ Medicine, Niigata Cancer Center Hospital, Niigata, Japan; ${ }^{3}$ Department of Thoracic Surgery, National Institute of Oncology-Semmelweis \\ University, Budapest, Hungary; ${ }^{4}$ National Koranyi Institute of Pulmonology, Budapest, Hungary; ${ }^{5}$ Department of Thoracic Surgery, Comprehensive \\ Cancer Center, Medical University of Vienna, Vienna, Austria; ${ }^{6}$ Department of Medical Oncology, Dana-Farber Cancer Institute, Boston, MA, USA \\ \#These authors contributed equally to this work. \\ Correspondence to: Professor Hongmei Wang. Department of Respiratory and Critical Care Medicine, The Affiliated Hospital of Qingdao University, \\ No. 59 Haier Road, Laoshan District, Qingdao 266000, China. Email: dor.whm@163.com.
}

\begin{abstract}
Asians who develop non-small cell lung cancer (NSCLC) have a chance of approximately $50 \%$ of harboring the epidermal growth factor receptor (EGFR) mutation. The G719X mutation in EGFR has 3 subtypes (i.e., G719A, G719C, or G719S), all of them being classified as uncommon EGFR mutations. The EGFR mutation G719X is most often associated with lung adenocarcinoma. Conversely, its occurrence in lung squamous cell carcinoma is rare. Its response to tyrosine kinase inhibitor (TKI) treatment remains unknown. A 50-year-old Asian male with no smoking history was admitted to our hospital (Affiliated Hospital of Qingdao University) with an irritating dry cough and 1 month of progressive dyspnea. The patient was diagnosed with lung squamous cell carcinoma (cT4N3M0, stage IIIC). Lung biopsy revealed the presence of EFGR G719X mutation. The patient received a tracheobronchial stent, targeted therapy, chemotherapy, seed implantation and radiotherapy, and survived for 25.4 months following diagnosis. It is crucial that gene mutation analysis is performed in non-smoking male squamous cell carcinoma patients. Compared to lung adenocarcinoma patients with rare G719X mutation, this lung squamous cell carcinoma patient with G719X-mutant tumor had a higher sensitivity to $2^{\text {nd }}$-generation EGFR-TKI treatment, but similar progression-free survival. Importantly, the patient clearly benefited from the used comprehensive treatment plan. This article seeks to shed light on the treatment of lung squamous cell carcinoma patients with the uncommon EGFR G719X mutation.
\end{abstract}

Keywords: Lung squamous cell carcinoma; G719X; gene mutation; case report

Submitted Sep 13, 2021. Accepted for publication Dec 23, 2021.

doi: 10.21037/atm-21-6653

View this article at: https://dx.doi.org/10.21037/atm-21-6653

\section{Introduction}

Lung cancer is the leading cause of cancer-related death and has the highest morbidity and mortality rates of malignant neoplasms worldwide. Non-small cell lung cancers (NSCLCs) account for more than $85 \%$ of all lung cancers (1), of which lung adenocarcinoma (LUAD) and lung squamous (LUSC) are the most common subtypes. NSCLCs can be divided into different clinically relevant subtypes. common mutated genes in Lung cancer is the epidermal growth factor receptor (EGFR), which is present in $27 \%$ of LUAD and $7 \%$ of LUSC (2). Squamous cell carcinomas account for about $20-30 \%$ of the prevalent NSCLCs (3). Mutations in the tyrosine kinase regions of the EGFR mainly occur in exons 18-21. Approximately $90 \%$ of mutations are related to exon 19 deletion and exon 21 L858R replacement (4). Meanwhile, approximately $10 \%$ of mutations, including G719X, L861Q, and S768I, are 
Table 1 A summary of the laboratory results

\begin{tabular}{lcc}
\hline Items & Admission & Reference interval \\
\hline HB $(\mathrm{g} / \mathrm{L})$ & 115 & $110-145$ \\
WBC $\left(\times 10^{9} / \mathrm{L}\right)$ & 10.42 & $5.5-9.5$ \\
PLT $\left(\times 10^{9} / \mathrm{L}\right)$ & 291 & $100-300$ \\
CEA $(\mathrm{ng} / \mathrm{mL})$ & 0.68 & $0-3.4$ \\
NSE $(\mathrm{ng} / \mathrm{mL})$ & 11.48 & $0-17$ \\
SCC $(\mathrm{ng} / \mathrm{mL})$ & 0.78 & $0-2.5$ \\
CA19-9 $(\mathrm{U} / \mathrm{mL})$ & 7.29 & $0-39$ \\
CA72-4 $(\mathrm{U} / \mathrm{mL})$ & 0.47 & $0-6.9$ \\
\hline
\end{tabular}

HB, hemoglobin; WBC, white blood cell; PLT, platelet; CEA, carcinoembryonic antigen; NSE, neuron specific enolase; SCC, squamous cell carcinoma; CA19-9, carbohydrate antigen199; CA72-4, carbohydrate antigen72-4.

uncommon or non-classical EGFR mutations (5). 20 exon insertion and complex mutations are the most frequently occurring mutations.

There is growing evidence that $2^{\text {nd }}$-generation tyrosine kinase inhibitor (TKI) treatment improves the outcomes of patients with rare EGFR mutations (6). However, there are no standard guidelines for treatment strategies in this population. The mutation status of the EGFR gene is closely related to the pathological type of lung cancer. With an occurrence rate of $50 \%$ (7), there was a previous pathological report of G719X with S768I mutation, but this case was early adenocarcinoma, which was actually detected in lung adenocarcinoma and is not uncommon (8), in addition, the case of severe squamous cell lung carcinoma harboured non-classical EGFR mutation has long OS after comprehensive therapy is the first to be reported as an individual case. EGFR mutations are the most common genetic mutations in Asian lung adenocarcinoma patients. Yet it accounts for only 3.6\% of lung squamous carcinoma (9). Therefore, it is very rare for lung squamous cell carcinoma patients to have single atypical EGFR mutations. In this paper, we report a case study of a lung squamous cell carcinoma patient with G719X gene mutation who had similar survival outcomes to lung adenocarcinoma patients with classical EGFR mutation. With this paper, we hope to provide insights into the treatment of rare G719X mutations in lung squamous carcinoma patients.

We present the following article in accordance with the CARE reporting checklist (available at https://dx.doi. org/10.21037/atm-21-6653).

\section{Case presentation}

A 50-year-old Asian male was admitted on 19 October 2018 with an irritating dry cough and 1 month of progressive dyspnea, but no expectoration, chest pain, hemoptysis, fever, nausea, vomiting, abdominal pain, or peripheral edema. The patient's mother had a history of lung cancer. There was no history of hypertension, diabetes mellitus, coronary artery disease, or stroke. Initially, the patient's vital signs were stable; the patient had a normal temperature $\left(36.5^{\circ} \mathrm{C}\right)$, a regular heart-beat $(75 / \mathrm{min})$ and breathing rate $(17 / \mathrm{min})$, normal blood pressure $(109 / 70 \mathrm{mmHg})$, and an oxygen saturation of $99 \%$ breathing room air. The physical examination did not reveal any abnormality in the lungs, heart or abdomen. The laboratory results for the routine blood and tumor markers are summarized in Table 1. Dynamic enhanced chest computed tomography (CT) showed a $16 \mathrm{~mm} \times 26 \mathrm{~mm}$ nodule located in the right upper lobe and compressed bronchi stenosis in the inferior segment of the trachea [see Figure 1 (A1,A2)] . To make a clinically accurate diagnosis, we performed CTguided mediastinal biopsy. However, the patient suffered from progressive chest tightness and shortness of breath and was unable to move or lie down. According to the electrocardiograph (ECG) monitoring results, the patients had a heart rate of $140-160 / \mathrm{min}$, his oxygen saturation decreased to $90-92 \%$ when the inhaled oxygen flow was $8 \mathrm{~L} / \mathrm{min}$. These results were thought to be related to his airway stenosis. We give symptomatic treatment, antitumor drug afatinib is commonly used to treat patients with the G719X mutation (10). The patient received targeted therapy of $40 \mathrm{mg} /$ day of afatinib in combination with chemotherapy.

The patient responded well to treatment and was discharged from the hospital (Affiliated Hospital of Qingdao University) after 14 days of hospitalization. Outpatient follow-up was continued, and after 1 month, the effect of treatment was assessed as partial response [see Figure $1(\mathrm{~B} 1, \mathrm{~B} 2)]$. We can see that the patient has a stent implantation. On October 29, 2018, an airway stent was implanted (metal straight tube bare stent) under local anesthesia. In January 2019, the patient was admitted to the Shanghai East Hospital where he underwent stent removal and mediastinal lymph node seed implantation. After 15.6 months, the patient was re-admitted to our hospital (Affiliated Hospital of Qingdao University) complaining of chest tightness and shortness of breath. Chest CT examinations indicated that the mediastinal lymph nodes were larger than before, which is indicative 

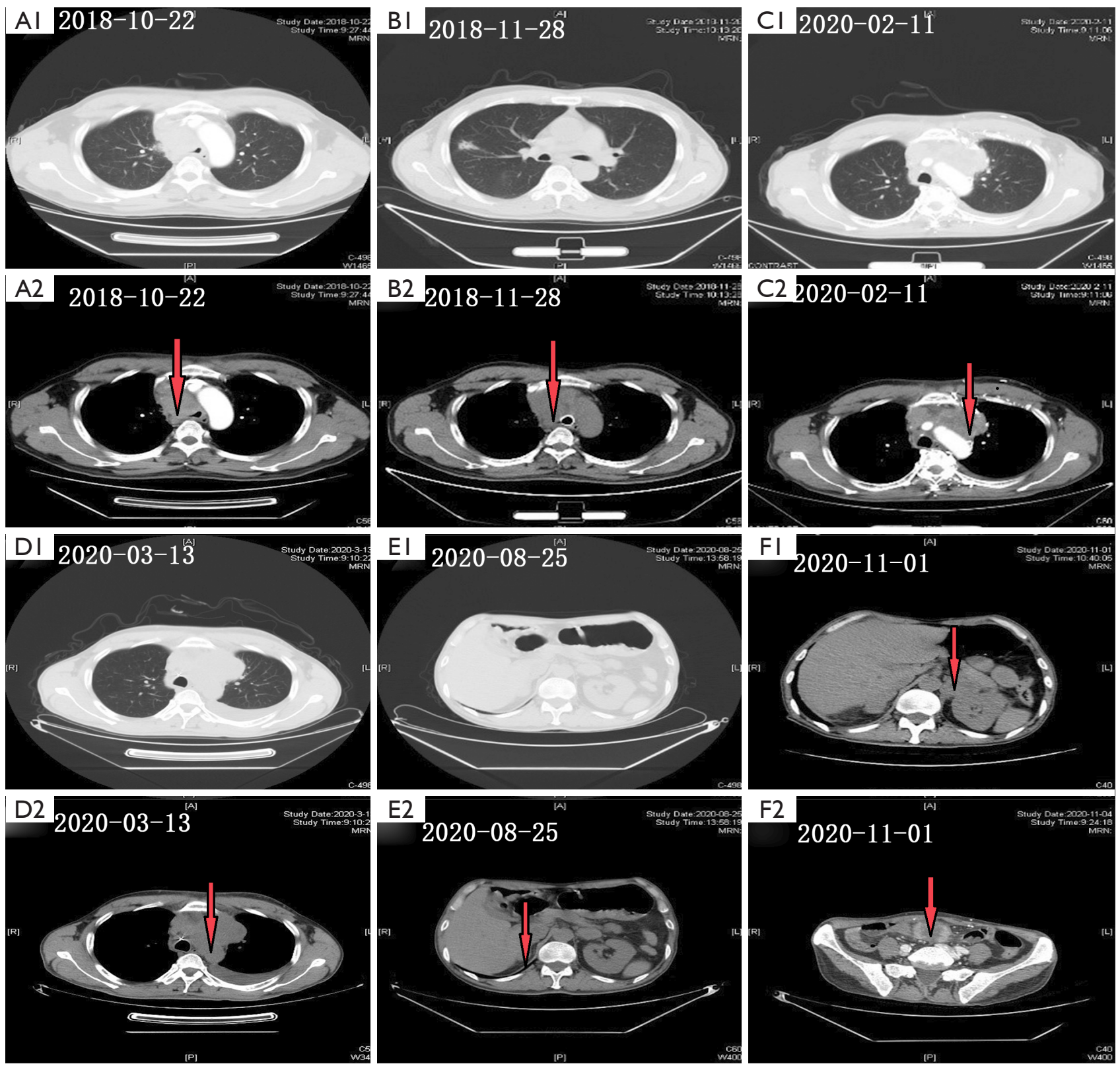

Figure 1 Imaging results of the computed tomography (CT) can during diagnosis and treatment at different time points. (A1,A2) Chest CT showed a $16 \mathrm{~mm} \times 26 \mathrm{~mm}$ nodule located in the right upper lobe and compressed bronchi stenosis in the inferior segment of the trachea. $(\mathrm{B} 1, \mathrm{~B} 2)$ After 1 months of Afatinib treatment, the two nodules were significantly smaller, and the efficacy was judged as a partial response. $(\mathrm{C} 1, \mathrm{C} 2)$ After 1 months of Afatinib treatment, the efficacy was judged as progressive disease (PD). (D1,D2) Chest CT revealed right pleural effusion metastasis. (E1,E2) Chest CT showed bilateral adrenal metastasis, the efficacy was again judged as PD. (F1) The abdominal CT showed mesenchymal pelvis metastasis (F2). The red arrow represents the tumor lesion or metastasis location.

of progressive disease [see Figure $1(\mathrm{C} 1, \mathrm{C} 2)]$. The $2^{\text {nd }}-$ line treatment (i.e., chemotherapy in combination with targeted therapy) was commenced. During the treatment, the symptoms of chest tightness and shortness of breath were relieved. However, after 2 cycles of chemotherapy, chest CT revealed right pleural effusion [see Figure 1 (D1,D2)] . Considering the disease progression, therapy with anlotinib combined with afatinib was started as the $3^{\text {rd }}$-line treatment. 

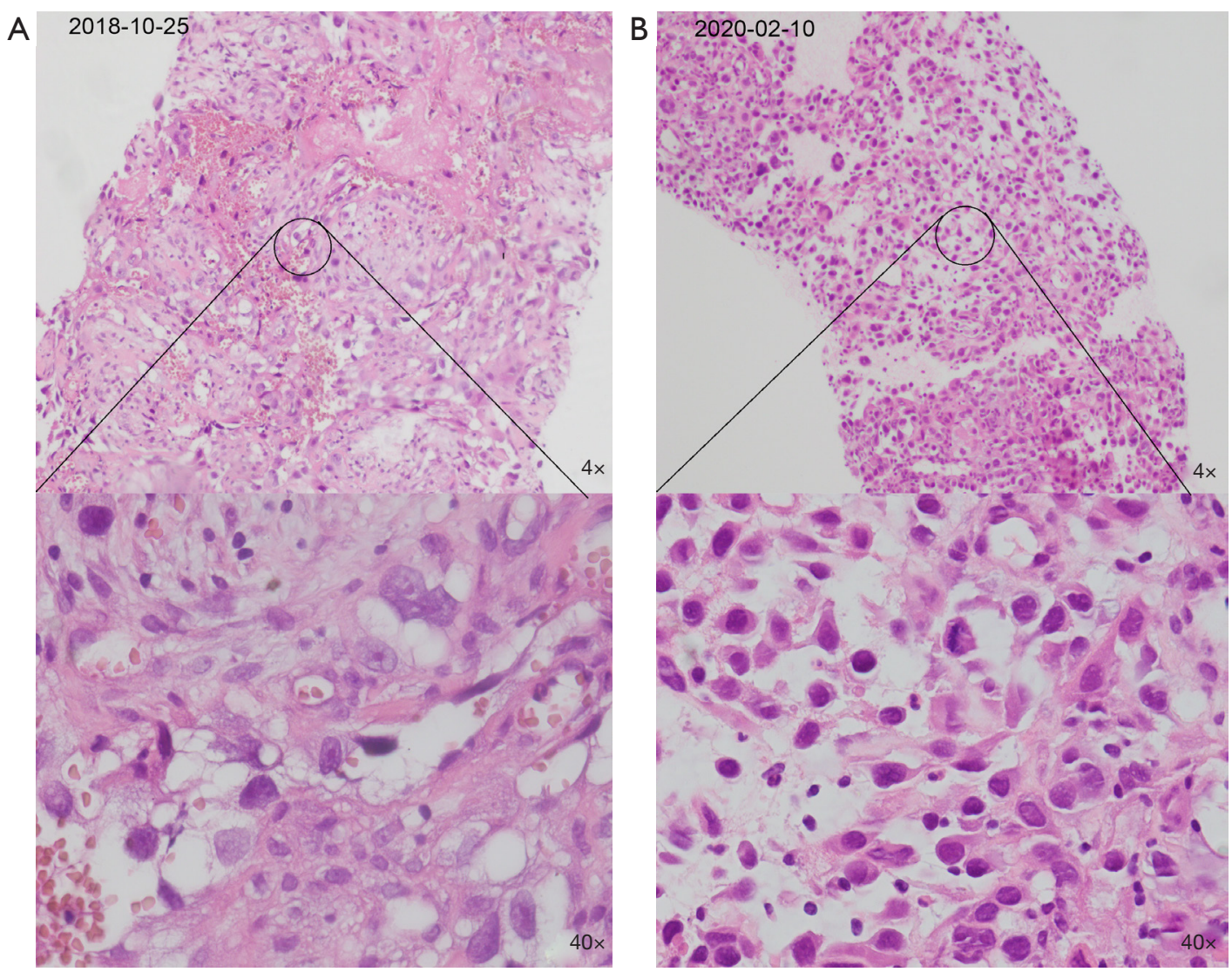

Figure 2 HE staining results of two lung biopsy tissues. (A) A puncture biopsy of mediastinal mass showed poorly differentiated carcinoma in proliferative fibrous connective tissue; (B) pathological puncture of lung tissue.

The patient was followed-up on a regular basis. On August 25, 2020, the patient experienced lumbar discomfort and chest CT showed bilateral adrenal metastasis [see Figure 1 $(\mathrm{E} 1, \mathrm{E} 2)]$. In the wake of disease progression, radiotherapy was applied. After 28 cycles of radiotherapy, the patient's condition showed a slight improvement, and the outpatient follow-up was continued.

On November 3, 2020, the patient was re-admitted with abdominal pain. Abdominal CT examination indicated bilateral adrenal metastasis progression [see Figure $1(\mathrm{~F} 1)$ ] and multiple mesenteric metastases in the mesenchymal pelvis [see Figure 1 (F2)], suggesting the accelerated progression of the disease. At this stage the patient's ECOG physical status score reached 4 points, in other words, the patient cannot carry on any selfcare, totally confined to bed or chair, so he had to choose palliative care and was given meperidine pain relief and nutritional support treatment.

We can see that the patient has a stent implantation.
On October 29, 2018, an airway stent was implanted (metal straight tube bare stent) under local anesthesia. A simultaneous biopsy indicated poorly differentiated infiltrating squamous cell carcinoma (see Figure $2 A$ ). The immunohistochemical examination revealed Cytokeratin [CK5/6(+)], p40(+), p63(+), Thyroid transcription factor [TTF-1(-)], NapsinA(-), Anaplastic Lymphoma KinaseD5F3 [ALK-D5F3(-)], ALK-D5F3-N(-), and EGFR exon 18 p.G719X. No distant metastasis or other enlarged lymph nodes were detected. Based on the results, the patient was diagnosed with lung squamous cell carcinoma (pT4N3M0, stage IIIC). At the first stage of disease progression, a biopsy of the lung tissue was performed, and the presence of the EGFR G719X mutation was again confirmed using histopathological examination (see Figure $2 B$ ). The patient rejected the recommended treatment of radiotherapy. The patient died on December 1, 2020, and, as shown in Figure 3, had an overall survival time of 25.4 months 


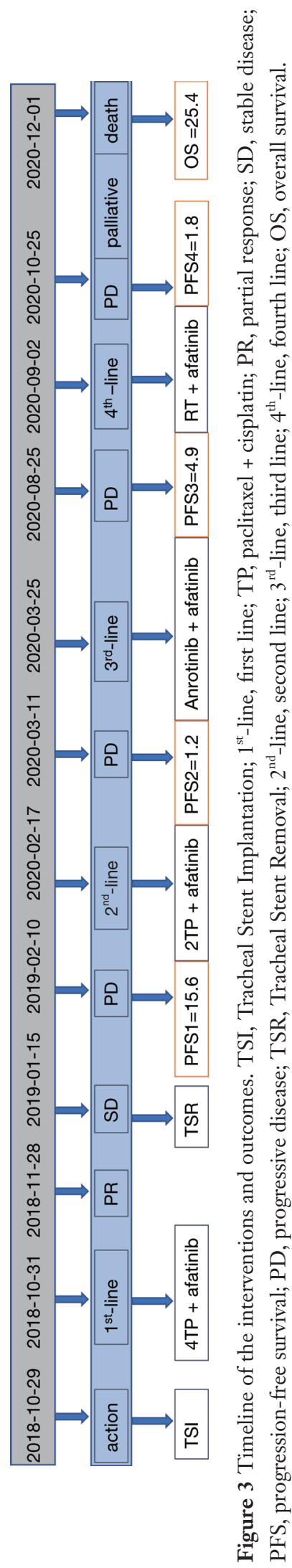

following diagnosis. Overall, patient compliance was fair. No unexpected serious adverse reactions were observed.

All procedures performed in this study involving human participants were in accordance with the ethical standards of the institutional and/or national research committee(s) and with the Helsinki Declaration (as revised in 2013). Written informed consent was obtained from the patient for publication of this case report and accompanying images. A copy of the written consent is available for review by the editorial office of this journal.

\section{IMDT discussion}

Several issues regarding the diagnosis and treatment of this patient were further discussed as follows

Question 1: Is there difference in the efficacy of EGFRTKI between lung squamous cell carcinoma with nonclassical mutation and adenocarcinoma? Expert opinion 1: Dr. Satoru Miura

There is few evidence regarding difference between squamous cell carcinoma and adenocarcinoma harboring uncommon EGFR mutations to the best my knowledge.

The polled analysis reported the efficacy of gefitinib for non-adenocarcinoma patients with common EGFR mutations was poor, with the response rate was $27 \%$ and the median progression free survival was 3.0 months (11). Based on these data, the efficacy of EGFR-TKI for lung squamous carcinoma with uncommon EGFR mutations may be limited.

\section{Expert opinion 2: Dr. Zsolt Megyesfalvi}

Initial studies identified several clinical parameters that were associated with a high response rate to EGFR TKIs and a relatively favorable prognosis. These include adenocarcinoma histology, female gender, non-smoker status, and Asian ethnicity. Accordingly, recent findings revealed that adenocarcinoma patients with classical EGFR mutations might indeed exhibit increased progressionfree survival (PFS) compared to those with EGFR-mutated squamous cell carcinoma (12). Nevertheless, due to the rarity of non-classical genetical alterations, the differences in the efficacy of EGFR TKIs in patients harboring uncommon EGFR mutations are rather controversial concerning the histological subtype. The current case report provides valuable insights into the therapeutic aspects of such patients, however further large-scale studies are needed to precisely answer the current question. Until then, all NSCLC patients with rare EGFR-TKI sensitizing 
mutations (such as G719X, S768I, and L861Q) should be followed-up and treated similarly (preferably with secondor third-generation TKIs) according to the contemporary guidelines.

\section{Expert opinion 3: Dr. Surein Arulananda}

The efficacy of EGFR TKI in lung squamous cell carcinoma regardless of mutation status is significantly lower in squamous cell carcinoma compared to adenocarcinoma as shown in a number of retrospective studies. We also know that the efficacy of EGFR TKI in lung adenocarcinoma with non-classical mutations such as G719X mutations is diminished, although the drug which appears to have the most promising activity is osimertinib based on a Phase II study (13). There is certainly data for afatinib in uncommon EGFR mutations as well (14). However there is a paucity of data for efficacy of EGFR TKI in squamous cell carcinoma with uncommon EGFR mutations, mainly because this is such a niche cohort of patients and most evidence for using EGFR TKI is from retrospective case studies. In this case study, platinum-taxane chemotherapy was used concurrently with afatinib, so it is difficult to delineate the degree to which afatinib contributed to the initial response, however as maintenance chemotherapy was not used and the total PFS was 15.6 months, we can only assume that afatinib contributed to this outcome. Having said that, I think it is reasonable to use an EGFR TKI in uncommon EGFR mutations upfront in patients regardless of tumour histology, but perhaps to be more judicious to switch to a chemotherapy $+/$ - immunotherapy strategy early if there is lack of tumour response on imaging or improvement in clinical symptoms.

\section{Question 2: Is it appropriate to intervene with radiotherapy when targeted therapy is effective? Expert opinion 1: Dr. Satoru Miura}

I think it depends on the patient's symptom and urgency of disease. In this case, the efficacy of EGFR-TKI is expected to be limited, so it is reasonable to perform the thoracic radiotherapy.

\section{Expert opinion 2: Dr. Zsolt Megyesfalvi}

EGFR regulates a number of interacting signaling pathways that can ultimately affect radiotherapy response (15). The concomitant use of monoclonal anti-EGFR antibody cetuximab with radiation therapy significantly improves local tumor control in different solid tumors. Nevertheless, due to its poor safety profile, combination cetuximab therapy did not enter the therapeutic armamentarium of NSCLC management. With regards to EGFR-TKIs, the combination of these targeted agents with radiation therapy has a strong biological rationale, as both first- and next-generation TKIs disrupt cell growth pathways and enhance the sensitivity of cells to radiation. Moreover, besides increasing radiosensitivity, EGFR-TKIs could also reduce TKI resistance. Radiation, on the other hand, may enhance the effectiveness of EGFR-TKIs via tumor cytoreduction (16). Therefore, the addition of radiotherapy to the already initiated EGFR-TKI therapy might be beneficial for patients with EGFR-mutated lung cancer. This statement is also further supported by the results of a recent phase II trial that showed favorable outcomes in NSCLC patients harboring EGFR mutations treated with concurrent EGFR-TKI plus thoracic radiotherapy (17). Of note, one should be aware that combining EGFR-TKIs with chemotherapy or radiation might lead to higher rates of severe toxicity-related complications such as interstitial pneumonitis.

\section{Expert opinion 3: Dr. Surein Arulananda}

Generally radiotherapy is determined up front based on the intent of treatment, i.e. curative versus palliative intent. In this situation, the patient had bulky Stage IIIC disease with significant symptoms and therefore a palliative approach was undertaken using systemic therapy. There is however emerging data with neoadjuvant systemic therapy for Stage III unresectable disease that is largely in early phase clinical trials and may in the future alter this paradigm. Seeing as that systemic therapy was effective and resulted in both tumor debulking with improvement in symptoms, there was not a major role to institute radiotherapy in this case, especially as the intent remained palliative.

\section{Question 3: What is the status of immunotherapy in lung squamous cell carcinoma with EGFR mutation after TKI treatment resistance? \\ Expert opinion 1: Dr. Satoru Miura}

The monotherapy of immune checkpoint inhibitor may be one of the option. However, there is no evidence. I consider the immunotherapy to be a treatment option after becoming resistance to standard chemotherapy, such as platinum doublet and docetaxel \pm ramucirumab.

\section{Expert opinion 2: Dr. Zsolt Megyesfalvi}

In general, NSCLC patients harboring EGFR mutations exhibit a poorer response to PD-1 or PD-L1 inhibitors than those bearing wild-type EGFR (regardless of histological subtype) (18). The exact pathomechanism behind the inferior response rates seen in these patients is yet to be elucidated. Nevertheless, previous investigations suggest 
that EGFR-mutated tumors have lower PD-L1 expression and tumor mutational burden (19). Clinicians should also bear in mind that the use of immune checkpoint inhibitors prior to or concurrent with EGFR-TKIs might increase the risk of pulmonary toxicity. Finally, it should be emphasized that in a considerable fraction of patients with EGFR mutations immunotherapy administration might lead to hyperprogressive disease and thus to poor outcomes. In case of EGFR-TKI resistance, the mechanisms of acquired resistance should be elucidated. If resistance to TKIs occurs due to wild-type EGFR amplification or loss of activating EGFR mutant gene, immunotherapy administration might be considered, but only in well-selected patients with high PD-L1 expression.

\section{Expert opinion 3: Dr. Surein Arulananda}

This is an interesting question because we know that the efficacy outcomes from immune checkpoint inhibitors in squamous cell carcinomas are generally favorable. However, we also know that lung adenocarcinomas with EGFR mutations tend to respond modestly at best to immune checkpoint inhibitors. The IMPower 150 study showed an improvement in efficacy outcomes in patients with EGFRmutant NSCLC resistant to TKI who were treated with the atezolizumab, bevacizumab and platinum-taxane regimen compared to bevacizumab plus chemotherapy. This was an exploratory analysis of a subset of patients within the trial and hence the results need to be considered carefully. Another interesting point is that EGFR mutant NSCLC with uncommon mutations have been shown to have a higher tumor mutational burden than common activating mutations (20). Therefore, although there is limited data for immune checkpoint inhibition in squamous cell lung cancer with EGFR mutations, I would consider that this is a strategy worth using after TKI resistance assuming there are no further secondary or tertiary EGFR mutations, such as the T790M mutation that can be targeted.

Question 4: Do multitarget antiangiogenic agents (MAA) combined with immunotherapy benefit patients with EGFR-TKI resistant lung squamous cell carcinoma? Expert opinion 1: Dr. Satoru Miura

Antiangiogenic agents expect to have a positive effect on immune microenvironment. Thus, I consider that the combination of such agents and immune checkpoint inhibitor is promising strategy to combat the "cold tumor" such as the EGFR-mutated tumor.

Expert opinion 2: Dr. Zsolt Megyesfalvi

As already elaborated in my previous answer (Answer \#3), immune checkpoint inhibitors should be administered with caution in patients with EGFR-TKI resistant squamous cell lung cancer and only after re-evaluating the tumoral EGFR mutational status (including the variant allele frequency) and PD-L1 expression. EGFR activation is associated with stimulated tumor angiogenesis and upregulated VEGF expression. Accordingly, antiangiogenic agents (such as bevacizumab) might be efficient in EGFR-mutated tumors and combining these therapeutic agents with EGFRTKIs might yield significant PFS benefits $(21,22)$. The use of multitarget antiangiogenic agents (MAA) should be also considered in the TKI-refractory cases. Combining immunotherapy with MAAs might as well be beneficial in certain patients since antiangiogenic therapy can elicit or enhance tumor immunity response, whereas reciprocally the immune system can support angiogenesis $(23,24)$. Therefore, combining MAAs and immunotherapy could be synergistic (at the cost of increased toxicities), however appropriate patient selection is crucial.

\section{Expert opinion 3: Dr. Surein Arulananda}

The biggest problem with antiangiogenic agents in squamous cell carcinoma is the high and unacceptable toxicity rates, in particular hemoptysis. In fact the vast majority of clinical trials using antiangiogenic agents have excluded patients with squamous cell lung carcinoma for this reason (25). Therefore, until safety data with a newer agent is presented, antiangiogenic agents, such as bevacizumab should not be considered for patients with squamous cell lung cancer.

\section{Discussion}

To the best of our knowledge, this is the first report on the clinical efficacy of afatinib in a lung squamous cell carcinomas patient with the uncommon G719X gene mutation. The G719X mutation occurs at exon 18 of the EGFR gene [glycine at position 719 is replaced by other amino acids such as alanine (G719A), cysteine (G719C) or serine (G719S)] (20). The proportion of G719X gene mutation in lung-line cell carcinoma is about $5 \%(8)$, and the proportion of mutation in LUSC is unknown. The G719X gene mutation at exon 18 responds to afatinib $(24,25)$, as the mutation increases the distance between L718 and G796, which in turn makes the binding pocket of adenosine triphosphate more open and more easily able to interact with EGFR-TKIs (23). The comprehensive results of a series of clinical trials showed that the objective response rate to afatinib in NSCLC patients with tumors 
harboring G719X gene mutation was $77.8 \%$, and the PFS period was 13.8 months $(26,27)$. The polled analysis reported the efficacy of gefitinib for non-adenocarcinoma patients with common EGFR mutations was poor, with the response rate was $27 \%$ and the median progression free survival was 3.0 months (11). However, the patient's PFS period in our case presentation was 15.6 months, which is consistent with in LUAD patients (14). The efficacy of target EGFR drugs in the case was higher than we originally expected_However, this observation needs to be further substantiated by prospective clinical trials.

Resistance to EGFR-TKIs appears frequently in advanced NSCLC patients with activating mutations in the EGFR gene (28). The positive T790M gene mutation is considered to be one of the most common cause of drug resistance concerning $1^{\text {st }}$ and $2^{\text {nd }}$ generation TKIs (29), causing approximately half of TKI-resistance (30). Importantly, G719X gene mutation often coexists with other mutations such as S768I and L861Q (10), or T790M gene mutation (31), and the co-existence of these mutations might contribute to drug resistance in patients with G719X mutations. Notably, a T790M gene test was performed on the mediastinal lymph node and peripheral blood of the patient, but no positive results were found. Therefore, it is likely that the previously presented lung squamous cell carcinoma patient had a single mutation (i.e., G719X gene mutation). Nevertheless, more research needs to be conducted to investigate whether single mutations exist in other lung squamous cell carcinoma patients with the G719X mutation. To overcome this issue, high-quality nextgeneration sequencing should be used to detect specific EGFR mutations in lung squamous cell carcinoma patients.

The efficacy outcomes from immune checkpoint inhibitors in squamous cell carcinomas are generally favourable (32). Nevertheless, previous investigations suggest that EGFR-mutated tumors have lower PD-L1 expression and tumor mutational burden (19). Programmed death-1 (PD-1) and its ligand (PD-L1) inhibitors are the representative drugs for immune checkpoint inhibitors (ICIs), and have led to breakthroughs in the immunotherapy of lung cancer patients. However, the efficacy of immunosuppressive agents has not been welldocumented in the treatment of patients with EGFR mutations (33). Some clinical studies have shown that the mutation status of EGFR is negatively correlated with PD-1 expression $(34,35)$, however, other reports suggest that EGFR mutations can actually upregulate PD-1 expression $(36,37)$ This observation might be of clinical importance, as traditional chemotherapy and anti-vascular treatment affect the expression level of PD-L (38,39). EGFR mutant NSCLC with uncommon mutations have been shown to have a higher tumour mutational burden (TMB) than common activating mutations (32), we would reserve immunotherapy as third line therapy after using platinum taxane chemotherapy, this is because there is limited data for using chemotherapy with anti-PD-1 therapy post 1 st line EGFR TKI. In fact, immunohistochemistry showed pD-L1 expression was $20 \%$. The patient could not receive immunotherapy due to the associated costs. However, a switch to immunotherapy before the patient's palliative treatment commenced may have prolonged the patient's survival.

It has been reported that $20-30 \%$ of lung cancer patients have malignant airway stenosis, which can cause breathing difficulties, weakened lung function, suffocation, and even death (40). The appearance of airway stenosis may be related to tumor obstruction in the cavity or external tumor compression (41). Surgery is not a viable option in such situations; thus, palliative interventions, such as stent implantation (42) and radioactive seed implantation (43), are used to destroy tumor tissues, reduce the degree of airway stenosis, and alleviate crises in the later stage. With regards to EGFR-TKIs, the combination of these targeted agents with radiation therapy has a strong biological rationale, as both first- and next-generation TKIs disrupt cell growth pathways and enhance the sensitivity of cells to radiation. Moreover, besides increasing radiosensitivity, EGFR-TKIs could also reduce TKI resistance. Radiation, on the other hand, may enhance the effectiveness of EGFR-TKIs by tumor cytoreduction (16). In patients with advanced adenocarcinoma, invasions of the mediastinum can cause fatal tracheoesophageal fistula after tracheal stent placement. This condition mostly occurs during rapid tumor ablation (44), such as radiotherapy and seed implantation. Thus, patients with advanced lung cancer receiving the local treatment of airway strictures need to be informed of the occurrence of this disease. At the end of his life, the patient was treated with multiple therapies, with multiple metastases, obstructive pneumonia, respiratory failure, and renal failure. In general, death from multifunctional organ failure is highly likely. As observed in the present case, timely local treatment can provide precious time for patients and achieved relatively long overall survival.

In summary, studies have shown that EGFR-TKI has variable effects on NSCLC patients with rare mutations. Due to the small number of lung squamous cell carcinoma 
patients with the G719X mutation, research on the efficacy of EGFR-TKIs in these patients is still inconclusive. Our case report provided further evidence of the diagnosis and treatment of an NSCLC patient with G719X gene mutation in a clinical setting. The underlying mechanisms of such mutations require immediate study. This will guide future efforts to formulate a reasonable and effective treatment plan based on the results of large-scale prospective clinical studies.

\section{Acknowledgments}

The authors appreciate the academic support from the AME Lung Cancer Collaborative Group.

Funding: This paper was supported by a grant from the Wu Jieping Medical Foundation (approval number: 320.6750.19094-29).

\section{Footnote}

Reporting Checklist: The authors have completed the CARE reporting checklist. Available at https://dx.doi. org/10.21037/atm-21-6653

Conflicts of Interest: All authors have completed the ICMJE uniform disclosure form (available at https://dx.doi. org/10.21037/atm-21-6653). SA reports that he receives payment from MSD, BMS, Roche, Astra Zeneca, and support from Astra Zeneca, Roche, MSD for attending meetings and participates in a Data Safety Monitoring Board or Advisory Board of Roche and Boehringer Ingelheim. The other authors have no conflicts of interest to declare.

Ethical Statement: The authors are accountable for all aspects of the work in ensuring that questions related to the accuracy or integrity of any part of the work are appropriately investigated and resolved. All procedures performed in this study involving human participants were in accordance with the ethical standards of the institutional and/or national research committee(s) and with the Helsinki Declaration (as revised in 2013). Written informed consent was obtained from the patient for publication of this case report and accompanying images. A copy of the written consent is available for review by the editorial office of this journal.

Open Access Statement: This is an Open Access article distributed in accordance with the Creative Commons Attribution-NonCommercial-NoDerivs 4.0 International License (CC BY-NC-ND 4.0), which permits the noncommercial replication and distribution of the article with the strict proviso that no changes or edits are made and the original work is properly cited (including links to both the formal publication through the relevant DOI and the license). See: https://creativecommons.org/licenses/by-nc-nd/4.0/.

\section{References}

1. Global Burden of Disease Cancer Collaboration; Fitzmaurice C, Dicker D, et al. The Global Burden of Cancer 2013. JAMA Oncol 2015;1:505-27.

2. Herbst RS, Morgensztern D, Boshoff C. The biology and management of non-small cell lung cancer. Nature 2018;553:446-54.

3. Cheng TY, Cramb SM, Baade PD, et al. The International Epidemiology of Lung Cancer: Latest Trends, Disparities, and Tumor Characteristics. J Thorac Oncol 2016;11:1653-71.

4. Zhang T, Wan B, Zhao Y, et al. Treatment of uncommon EGFR mutations in non-small cell lung cancer: new evidence and treatment. Transl Lung Cancer Res 2019;8:302-16.

5. Evans M, O'Sullivan B, Smith M, et al. Large-Scale EGFR Mutation Testing in Clinical Practice: Analysis of a Series of 18,920 Non-Small Cell Lung Cancer Cases. Pathol Oncol Res 2019;25:1401-9.

6. Wu YL, Hirsh V, Sequist LV, et al. Does EGFR Mutation Type Influence Patient-Reported Outcomes in Patients with Advanced EGFR Mutation-Positive Non-Small-Cell Lung Cancer? Analysis of Two Large, Phase III Studies Comparing Afatinib with Chemotherapy (LUX-Lung 3 and LUX-Lung 6). Patient 2018;11:131-41.

7. Wu YL, Zhong WZ, Li LY, et al. Epidermal growth factor receptor mutations and their correlation with gefitinib therapy in patients with non-small cell lung cancer: a meta-analysis based on updated individual patient data from six medical centers in mainland China. J Thorac Oncol 2007;2:430-9.

8. Cai Y, Wang Y, Sun J, et al. Successful treatment of a patient with NSCLC carrying uncommon compound EGFR G719X and S768I mutations using osimertinib: A case report. J Int Med Res 2020;48:300060520928793.

9. Tate JG, Bamford S, Jubb HC, et al. COSMIC: the Catalogue Of Somatic Mutations In Cancer. Nucleic Acids Res 2019;47:D941-7. 
10. Yang JC, Sequist LV, Geater SL, et al. Clinical activity of afatinib in patients with advanced non-small-cell lung cancer harbouring uncommon EGFR mutations: a combined post-hoc analysis of LUX-Lung 2, LUX-Lung 3, and LUX-Lung 6. Lancet Oncol 2015;16:830-8.

11. Shukuya T, Takahashi T, Kaira R, et al. Efficacy of gefitinib for non-adenocarcinoma non-small-cell lung cancer patients harboring epidermal growth factor receptor mutations: a pooled analysis of published reports. Cancer Sci 2011;102:1032-7.

12. Jin R, Peng L, Shou J, et al. EGFR-Mutated Squamous Cell Lung Cancer and Its Association With Outcomes. Front Oncol 2021;11:680804.

13. Cho JH, Lim SH, An HJ, et al. Osimertinib for Patients With Non-Small-Cell Lung Cancer Harboring Uncommon EGFR Mutations: A Multicenter, OpenLabel, Phase II Trial (KCSG-LU15-09). J Clin Oncol 2020;38:488-95.

14. Yang JC, Schuler M, Popat S, et al. Afatinib for the Treatment of NSCLC Harboring Uncommon EGFR Mutations: A Database of 693 Cases. J Thorac Oncol 2020;15:803-15.

15. Sacco PC, Maione P, Rossi A, et al. Combination of radiotherapy and targeted therapies in the treatment of locally advanced non-small cell lung cancer. Target Oncol 2011;6:171-80.

16. Komaki R, Allen PK, Wei X, et al. Adding Erlotinib to Chemoradiation Improves Overall Survival but Not Progression-Free Survival in Stage III Non-Small Cell Lung Cancer. Int J Radiat Oncol Biol Phys 2015;92:317-24.

17. Zheng L, Wang Y, Xu Z, et al. Concurrent EGFR-TKI and Thoracic Radiotherapy as First-Line Treatment for Stage IV Non-Small Cell Lung Cancer Harboring EGFR Active Mutations. Oncologist 2019;24:1031-e612.

18. Gainor JF, Shaw AT, Sequist LV, et al. EGFR Mutations and ALK Rearrangements Are Associated with Low Response Rates to PD-1 Pathway Blockade in Non-Small Cell Lung Cancer: A Retrospective Analysis. Clin Cancer Res 2016;22:4585-93.

19. To KKW, Fong W, Cho WCS. Immunotherapy in Treating EGFR-Mutant Lung Cancer: Current Challenges and New Strategies. Front Oncol 2021;11:635007.

20. Li K, Yang M, Liang N, et al. Determining EGFRTKI sensitivity of G719X and other uncommon EGFR mutations in non-small cell lung cancer: Perplexity and solution (Review). Oncol Rep 2017;37:1347-58.

21. Han B, Tjulandin S, Hagiwara K, et al. EGFR mutation prevalence in Asia-Pacific and Russian patients with advanced NSCLC of adenocarcinoma and nonadenocarcinoma histology: The IGNITE study. Lung Cancer 2017;113:37-44.

22. Beau-Faller M, Prim N, Ruppert AM, et al. Rare EGFR exon 18 and exon 20 mutations in non-small-cell lung cancer on 10117 patients: a multicentre observational study by the French ERMETIC-IFCT network. Ann Oncol 2014;25:126-31.

23. Lynch TJ, Bell DW, Sordella R, et al. Activating mutations in the epidermal growth factor receptor underlying responsiveness of non-small-cell lung cancer to gefitinib. N Engl J Med 2004;350:2129-39.

24. Otsuka T, Mori M, Yano Y, et al. Effectiveness of Tyrosine Kinase Inhibitors in Japanese Patients with Non-small Cell Lung Cancer Harboring Minor Epidermal Growth Factor Receptor Mutations: Results from a Multicenter Retrospective Study (HANSHIN Oncology Group 0212). Anticancer Res 2015;35:3885-91.

25. Leduc C, Merlio JP, Besse B, et al. Clinical and molecular characteristics of non-small-cell lung cancer (NSCLC) harboring EGFR mutation: results of the nationwide French Cooperative Thoracic Intergroup (IFCT) program. Ann Oncol 2017;28:2715-24.

26. Yang JC, Shih JY, Su WC, et al. Afatinib for patients with lung adenocarcinoma and epidermal growth factor receptor mutations (LUX-Lung 2): a phase 2 trial. Lancet Oncol 2012;13:539-48.

27. Yang JC, Wu YL, Schuler M, et al. Afatinib versus cisplatin-based chemotherapy for EGFR mutation-positive lung adenocarcinoma (LUX-Lung 3 and LUX-Lung 6): analysis of overall survival data from two randomised, phase 3 trials. Lancet Oncol 2015;16:141-51.

28. Maemondo M, Inoue A, Kobayashi K, et al. Gefitinib or chemotherapy for non-small-cell lung cancer with mutated EGFR. N Engl J Med 2010;362:2380-8.

29. Sharma SV, Bell DW, Settleman J, et al. Epidermal growth factor receptor mutations in lung cancer. Nat Rev Cancer 2007;7:169-81.

30. Yu HA, Arcila ME, Rekhtman N, et al. Analysis of tumor specimens at the time of acquired resistance to EGFR-TKI therapy in 155 patients with EGFR-mutant lung cancers. Clin Cancer Res 2013;19:2240-7.

31. Ancevski Hunter K, Friedland DM, Villaruz LC, et al. First-Line Osimertinib in Patients with Treatment-Naive Somatic or Germline EGFR T790M-Mutant Metastatic NSCLC. J Thorac Oncol 2018;13:e3-5.

32. Chen K, Pan G, Cheng G, et al. Immune microenvironment features and efficacy of PD-1/PD- 
L1 blockade in non-small cell lung cancer patients with EGFR or HER2 exon 20 insertions. Thorac Cancer 2021;12:218-26.

33. Herbst RS, Baas P, Kim DW, et al. Pembrolizumab versus docetaxel for previously treated, PD-L1-positive, advanced non-small-cell lung cancer (KEYNOTE-010): a randomised controlled trial. Lancet 2016;387:1540-50.

34. Lee SE, Kim YJ, Sung M, et al. Association with PD-L1 Expression and Clinicopathological Features in 1000 Lung Cancers: A Large Single-Institution Study of Surgically Resected Lung Cancers with a High Prevalence of EGFR Mutation. Int J Mol Sci 2019;20:4794.

35. Takada K, Toyokawa G, Tagawa T, et al. PD-L1 expression according to the EGFR status in primary lung adenocarcinoma. Lung Cancer 2018;116:1-6.

36. Song Z, Yu X, Cheng G, et al. Programmed death-ligand 1 expression associated with molecular characteristics in surgically resected lung adenocarcinoma. J Transl Med 2016;14:188.

37. Tang $Y$, Fang W, Zhang $Y$, et al. The association between PD-L1 and EGFR status and the prognostic value of PDL1 in advanced non-small cell lung cancer patients treated with EGFR-TKIs. Oncotarget 2015;6:14209-19.

38. Chen Y, Liu YC, Sung YC, et al. Overcoming sorafenib

Cite this article as: $\mathrm{Bi} \mathrm{H}$, Ren D, Wu J, Ding X, Guo C, Miura S, Megyesfalvi Z, Arulananda S, Wang H. Lung squamous cell carcinoma with rare epidermal growth factor receptor mutation G719X: a case report and literature review. Ann Transl Med 2021;9(24):1805. doi: 10.21037/atm-21-6653 evasion in hepatocellular carcinoma using CXCR4targeted nanoparticles to co-deliver MEK-inhibitors. Sci Rep 2017;7:44123.

39. Guo L, Song P, Xue X, et al. Variation of Programmed Death Ligand 1 Expression After Platinum-based Neoadjuvant Chemotherapy in Lung Cancer. J Immunother 2019;42:215-20.

40. Ernst A, Feller-Kopman D, Becker HD, et al. Central airway obstruction. Am J Respir Crit Care Med 2004;169:1278-97.

41. Mahajan AK, Ibrahim O, Perez R, et al. Electrosurgical and Laser Therapy Tools for the Treatment of Malignant Central Airway Obstructions. Chest 2020;157:446-53.

42. Ost DE, Ernst A, Grosu HB, et al. Complications Following Therapeutic Bronchoscopy for Malignant Central Airway Obstruction: Results of the AQuIRE Registry. Chest 2015;148:450-71.

43. Mudambi L, Miller R, Eapen GA. Malignant central airway obstruction. J Thorac Dis 2017;9:S1087-110.

44. Mizuguchi S, Takahama M, Nakajima R, et al. Rapid Progression of Tracheoesophageal Fistula Caused by Immunotherapy Administered after Tracheal Stent Placement. Biomed Hub 2019;4:1-5. 